\title{
State of the Art in Prosthetic Wrists: Commercial and Research Devices
}

\author{
Neil M. Bajaj, Adam J. Spiers and Aaron M. Dollar \\ Department of Mechanical Engineering and Materials Science \\ Yale University \\ New Haven, CT 06511 \\ neil.bajaj@yale.edu, adam.spiers@yale.edu, aaron.dollar@yale.edu
}

\begin{abstract}
The human wrist contributes greatly to hand mobility and manipulation capabilities in healthy individuals, but both the commercial and research domains have often overlooked prosthetic wrists in favor of terminal device development. In this paper, we review the current state of the art of in a wide variety of passive, body powered and active wrists from both the prosthetics industry and research community. We primarily focus on the mechanical design and kinematic arrangement of these systems, giving details of articulation methods and specifications where possible. Among other take-aways, the review shows that very few powered wrists are available commercially, all of which are single-DOF, that multi-DOF wrist designs are most often serial chain systems, and that there seems to be opportunities for the development of body-powered wrist devices or wrists with a parallel kinematic architecture. Additionally, of the three DOF of the human wrist, radial/ulnar deviation is least commonly implemented in hardware.
\end{abstract}

\section{Keywords-Wrist, Prosthesis, Prosthetics, Hand, Arm}

\section{INTRODUCTION}

In the United States alone, there are approximately 41,000 upper limb amputees [1]. Continuing efforts in upper limb prosthetics have led to a variety of high dexterity terminal devices (TDs) that strive to imitate the function of the human hand. However, relatively little work has been done in the development of prosthetic wrists, despite the significant role the wrist plays in manipulation tasks [2], [3]. Recent investigations have debated whether increased dexterity of prosthetic wrists may serve amputees better than highly dexterous hands [4]. Among benefits including better mobility of the TD, such dexterous wrists could also reducing longer term complications from compensatory body motions [4].

In this paper, we conduct a review of wrist prostheses in both the prosthetics industry (commercial devices) as well as in the academic research community. Note that the wrist prostheses investigated here are not arthroplasty (joint replacement) implants for non-amputated individuals, but rather external devices for upper-limb amputees. This review will focus primarily on the hardware design of prosthetic wrist devices, as opposed to work related to issues such as control methodologies. We examine passive, body powered, and active (electrically-powered) wrists, and further classify the devices by their mechanical topology (joint types and arrangement) and relationship to natural wrist DOF.

We begin with an overview of the human wrist to provide a basis on which to consider the prostheses. This is followed by a brief introduction to mechanism terminology and topology and a summary of common issues with prosthetic wrists is given. We then conduct the review of prosthetic wrist devices. This includes a table of mechanical specifications for the few devices whose properties were available. Finally, we discuss the findings of the review and draw conclusions on the possible directions of future wrist prosthesis development.

\section{HUMAN WRIST MOTION CAPABILITY}

The healthy human wrist is capable of three degrees of freedom (DOF). These DOFs are pronation/supination, flexion/extension, and radial/ulnar deviation (Figure ). Radial/ulnar deviation is sometimes called abduction/adduction (respectively), and pronation/supination may be called wrist rotation. Note that each DOF is a paired set of motions (e.g. flexion/extension), involving positive and negative rotations from the neutral pose about the same axis. Here after, when only one of the motions per axes is named, we refer to both motions (e.g. pronation implies both pronation and supination).

It should be noted that pronation/supination does not occur at the carpal bones of the wrist, but actually occurs within the forearm, as the radius crosses over the ulna in a twisting motion when moving from supinated to pronated positions. It should also be noted that motion originating in the carpal bones (flexion/extension and radial/ulnar deviation) is coupled, meaning that motion in one DOF limits motion in the other (e.g. the amount of radial deviation is reduced when fully extended than from the neutral position). 

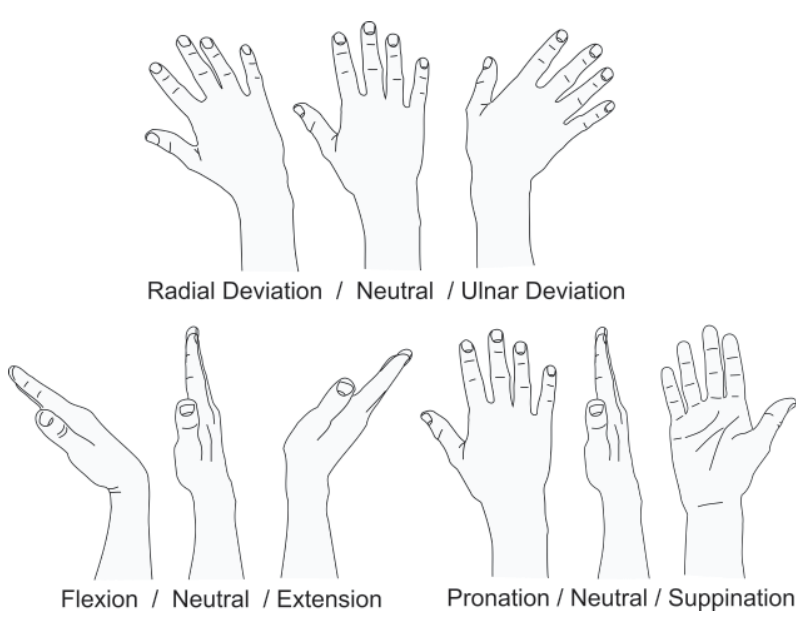

Figure 1: Three degrees of freedom (DOF) of the healthy human wrist.

For an unaffected human wrist, the maximal ranges of each DOF fall within the bounds of $76^{\circ} / 85^{\circ}, 75^{\circ} / 75^{\circ}$, and $20^{\circ} / 45^{\circ}$ for pronation/supination, flexion/extension, and radial/ulnar deviation, respectively [5]-[7]. Various groups have studied the range of motion (ROM) of the various DOF of the human wrist while performing Activities of Daily Living (ADLs). These investigations have generated a variety of ROM that fall within the bounds of $65^{\circ} / 77^{\circ}$, $50^{\circ} / 70^{\circ}$, $\quad 18^{\circ} / 40^{\circ}$ for pronation/supination, flexion/extension, and radial/ulnar deviation, respectively [8]-[11]. Wrist pronation torque ranges from 6-10 Nm [12], and flexion torque ranges from 8-14 $\mathrm{Nm}$ [13].

\section{PROSTHETIC WRIST CHARACTERISTICS}

In the following sections of this paper we present a review of 62 prosthetic wrists. For ease of navigation, these devices will be categorized based on articulation method, DOFs, joint type(s) and mechanism type(s). In this section we define these categories of classification. For a limited number of devices it was possible to locate (in literature) or directly measure, physical specifications, such as weight, length and (for active systems) torque. These values are provided in Table 1 .

\section{A. Articulation Method}

The function of a prosthetic wrist is to enable reorientation of a TD relative to the forearm. Articulation is achieved via Passive, Body Powered, or Active methods.

In a passive wrist the user manually adjusts wrist and terminal device (TD) position, either through their contralateral arm, body and/or environmental features [14]. For example, a terminal device may be clamped between the hip and table edge to enable re-orientation. Body powered devices utilize motion and accompanying forces in other parts of the user's body to achieve motion of a prosthetic device. The user typically wears a shoulder harness with an attached Bowden cable to transmit force from the shoulder to the prosthesis. Most commonly this is used to achieve opening/closing of a terminal device, such as a Hosmer Hook [15], but body powered mechanisms have also been applied to other prosthetic components (as

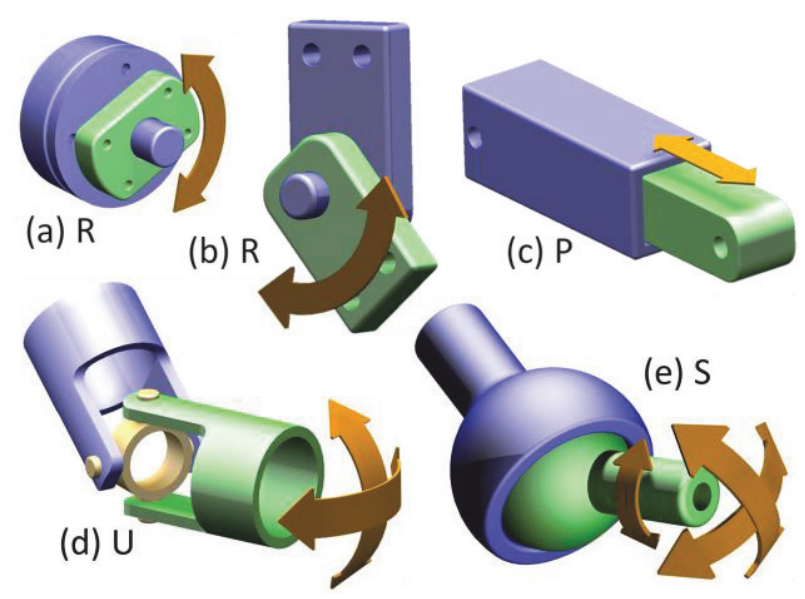

Figure 2: Types of Mechanical joints: (a),(b) Revolute (1DOF, R) either arranged as a rotator (a) or flexor (b). (c) Prismatic (1DOF, P) capable or linear motion. (d) Universal (2DOF - U) and (e) Spherical

will be discussed later). In active devices, an actuator, such as an electric motor, is used to generate force and motion in the prosthesis. Such devices are typically part of myoelectric systems. Note that a prosthetic device may have an actuator in it, but will not be considered an active mechanism in this paper if the actuator does not exert control over at least 1 DOF of the wrist. For example, an active hand with a passive wrist would not be considered an active wrist in this in this review.

\section{B. Joint Types}

For the purposes of this paper, we consider 4 types of joints, which may be used as standalone elements or form a serial chain. The 4 types of joints a chain are revolute (R), prismatic (P), universal (U), and spherical (S) joints. These 4 joints are schematically shown in Fig. 2. Combinations of joint are used to create mechanisms.

\section{Mechanism Type}

A mechanism may be a serial, parallel, or hybrid depending on the relationship of its linkage(s).

A serial mechanism is a sequential connection of joints and links. For example a 2R mechanism (also known as an RR mechanism) is a $2 \mathrm{DOF}$ system composed of two revolute joints located sequentially after one another.

A parallel mechanism consists of 2 or more serial chains which span from a common base to a common distal platform, which in this case is generally the TD.

A hybrid mechanism, finally, is a combination of serial and parallel mechanisms. This could be, for example, a parallel mechanism in series with a serial mechanism, so that the base of one mechanism may not be fixed in space or the distal part of one mechanism may not terminate in the end effector. An example of a hybrid mechanisms occurs in the PKM Tricept T805 industrial robot, where a 3DOF parallel mechanism terminates in a 3DOF serial wrist [16]. 


\section{Common issues with Wrist Prostheses}

Major issues that limit the application of wrist prostheses are the additional weight and limb length resulting from the integration of a wrist unit into an existing socket (worn over the residual limb) and terminal device arrangement. Although additional weight of a prosthetic can lead to fatigue and discomfort, excessive length can create a discrepancy between limbs that impacts co-ordination and aesthetics. Upper limb prosthetics are already subject to abandonment due to these reasons, even without the inclusion of a wrist [17].

Though forearm socket length may be reduced to accommodate the additional length of a wrist, this is only applicable to amputees with relatively short residual limbs (proximal amputation). It is essential to make the distinction between a wrist disarticulation amputee vs. a transradial (forearm) and other type of upper limb amputee when preparing a prosthesis. In the former case, a long residual limb prevents the prosthesis from occupying any forearm volume, whereas in the latter cases, a portion of the forearm socket is available to house components of the prosthesis (such as the battery in myoelectric devices).

\section{Passive Wrist Prostheses}

The review will first cover concepts and nomenclature before discussing passive, body powered and then active wrist prostheses. The wrists are arranged in each section in order of increasing DOF and linkage configuration.

\section{A. Passive Wrist Articulation Techniques}

As previously mentioned, passive devices mainly rely on manual repositioning of the wrist by a user's unaffected (contralateral) hand. For bi-lateral amputees, such repositioning can be difficult and available environmental features may be used in lieu of a contralateral hand.

To prevent wrist motion after positioning, passive devices make use of either friction clutches or locking mechanisms. A friction clutch is a simple mechanism that relies on the friction between components of a joint in order to prevent joint motion. The joint will then only move if a torque is provided that overcomes the holding friction. Generally, the holding friction may be adjusted by means of set screws as in [15], [18]-[24] and Fig. 1.a and 3.c. Whereas friction-clutch based devices may be positioned within a continuous range, locking mechanisms enable positioning of the joint to a limited number of discrete angles. The joint is then locked in the position by means of a sprung catch, pin or button [15], [25]-[32]. This permits the joint to remain immobile in the presence of relatively large torques. Fig. 1.b illustrates the passive HD Friction Wrist, with both friction clutch for pronation and locking mechanism for flexion. Some passive wrist prostheses make use of body powered interfaces to toggle the engagement of the locking mechanism (Fig 3.e). These systems will be described further in Section V. Note that the use of friction clutch mechanisms as flexion unit may lead to problems with bodypowered terminal devices. Cable tension applied to the terminal device for articulation could easily overcome the friction threshold for wrist motion, leading to unwanted reorientation. A joint locking mechanism overcomes this issue.

Elastic elements, such as torsional springs, have also been used to enable articulation of wrist mechanisms. Elastic elements return the wrist to a biased flexion point when external force is removed. Such flexibility imparts a degree of passive and adaptive behavior during interaction with partially constrained objects or during bi-manual tasks. One use scenario is bi-manual gripping of a bike's handlebars, whose position and orientation is controlled by a (healthy) contralateral hand and constrained by the central pivot of the bike frame. Elastic elements in this scenario would adapt the orientation of the wrist to the changing orientation of the handlebars to allow bimanual grasping, improving rider stability. Another example may be rowing a boat [33], where complex oar orientation is matched by an elastic wrist. A multi-DOF wrist incorporating torsional springs in a universal joint is illustrated in Fig. 1.d.

\section{B. Review of Passive Wrists}

\section{1) Passive Single $\mathrm{DOF}$}

Single DOF wrists can be compact and mechanically simple, and thus have largely been the status quo of prosthetic wrist usage for the last 75 years.

\section{a) Rotators}

The most simple passive wrists consist of a single, inline rotational joint (Figure .a) to allow terminal device pronation [15], [18]-[22], [25]-[29]. An example of such a rotator, the Hosmer Friction Wrist, is shown in Fig. 1.a, which also incorporates a friction clutch. . Hosmer Dorrance [15] (here after, $H D$ ) offers a variety of commercial passive rotators with the same functionality as many of those listed in the following descriptions (Fig. 3a, 3b). Standalone friction clutch examples are given [18], [19]. The devices in [20], [21] are integrated into a transhumeral prosthesis and terminal device, respectively. Locking mechanism examples are given in [22], [25]. The mechanisms in [26], [27] require the user to turn an external shell relative to the socket to impart pronation, otherwise the joint remains locked. Similarly, devices presented in [28], [29] are nonbackdriveable, meaning that forces and torques on the TD do not change the position of the wrist, with only torques applied to a collar of the wrist unit itself rotates the TD. Some of these rotators [25], [28], [29] also serve as 'quick disconnects (QDs)', allowing the user to easily remove and change their TD.

\section{b) Flexors}

An alternative use of a single rotational joint is to enable wrist flexion, as in [15], [30]-[32]. All of these devices include a locking function as described in Section IV.A. Commercial flexion products include the HD Sierra Wrist [15] and the Otto Bock (OB) MyoWrist [31], [32].

\section{2) Passive $2 R$ or $U$ Mechanisms}

The use of 2 rotational $(\mathrm{R})$ joints, orthogonally placed in series creates the same mechanism as a Universal (U) joint (Figure .c.). The HD 4-Function Wrist is simply a combination of the company's Rotation Wrist and Sierra 


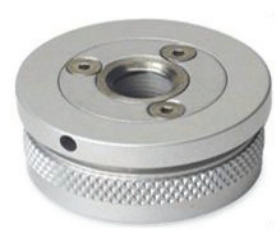

(a) $1 \mathrm{R}$

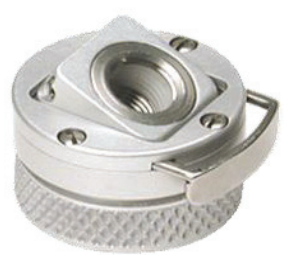

(b) $2 \mathrm{R}$

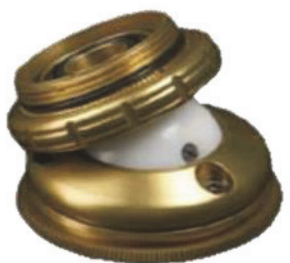

(c) U*

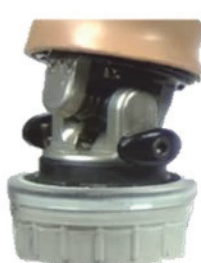

(d) RU

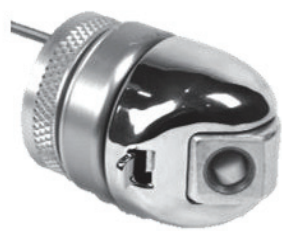

(e) $2 \mathrm{R}$

Fig. 1: Passive Wrist Mechanisms of (a) Hosmer Dorrance (HD) Friction Wrist (R) [15], (b) HD FW Flexion Friction Wrist (2R) [15], (c) Otto Bock Myolino

Wrist (U* - an S joint with prevented rotation) [24] and (d) Motion Control MultiFlex Wrist (RU)[37]. (e) Body Powered (2R) HD 4- Function Wrist [15].

Wrist products [15] (Fig 3.e.). This enables both pronation and flexion in one mechanism. The 4-Function Wrist utilizes a body powered pronation lock, which will be discussed further in Section V. Similarly, two locking flexion units were placed in series with a $90^{\circ}$ offset in [34], resulting in lockable flexion and radially deviation. A downside to this approach is the excessive length of the resulting wrists (see Section III.D for length issues).

The Motion Control (MC) Flexion Wrist [33] couples a friction based wrist rotator with wrist flexor containing elastic elements (torsional springs). These springs afford the benefits described in Section IV.A. Similarly, the wrist described in [35] utilizes a bevel gear differential to achieve flexion and radial deviation, with elastic elements implemented to bias the TD to a neutral position.

The same multi-DOF dexterity as $2 \mathrm{R}$ approaches may be achieved in a more compact form via modified spherical joints, such as those used in the LTI OmniWrist [23] (Fig 3.c) or $O B$ MyoLino Wrist [24]. By constraining the roll axis of a spherical joint via a circumferential groove, the $3 \mathrm{DOF}$ mechanism is reduced to a $2 \mathrm{DOF} \mathrm{U}$ joint, allowing flexion and radial deviation. These mechanisms rely on friction clutches, which may be individually adjusted for each DOF [24]. As discussed in Section IV.A, these wrists are not recommended for use with cable actuated TDs.

The wrist described in [36] is composed of a single $U$ joint to allow for flexion and radial deviation. This wrist is part of a powered TD, which also uses the $U$ joint to transmit power from a motor located in the forearm to the hand for grasping, This is accomplished by rotation of the entire $\mathrm{U}$ joint itself, which does not affect wrist motion.

\section{3) Passive RU Mechanisms}

The arrangement of joints in an RU wrist resembles the skeletal structure of the human wrist, with the carpal bones (analogous to a $\mathrm{U}$ joint) distal to the radial and ulnar bones (analogous to an $\mathrm{R}$ joint). In terms of passive serial RU wrists, the MC Multiflex [37] utilizes a pronation unit in series with a universal joint. Flexion and radial deviation occur distally, at the $\mathrm{U}$ joint. Similar to the $M C$ Flexion Wrist [33], both DOFs of the universal joint are biased to a neutral position via elastic elements. There is only a length increase of $6 \mathrm{~mm}$ between the 2DOF 2R Flexion Wrist and the 3DOF RU Multi-Flex Wrist.

\section{4) Passive Spherical $S$ Wrists}

Spherical (S) joints (Figure .e) offer 3 DOF with high compactness due to the absence of a serial chain. It is necessary that the ball of the spherical joint is partially encapsulated by the socket. This leads to a limited range of motion in the non-pronating DOF. An additional disadvantage is lack of stability, as locking mechanisms are not available for $\mathrm{S}$ joints, so friction clutches must be relied upon. A passively spherical wrist permitted positioning of an experimental hand in [38]. Here, the hollow center of the spherical joint allows actuated tendons to pass from the forearm to the hand. In [39], a hand is placed atop a spherical wrist and passively prepositioned before interaction with other objects.

\section{BOdy POWERED Wrist PROSTHESES}

Body powered interfaces are often used for the articulation of prosthetic terminal devices. By wearing a body harness, an amputee may use the motion of their arm or shoulder to exert tension on a Bowden cable, which terminates on the mechanism of some prosthetic device.

There are a limited number of wrist prostheses with body powered interfaces, despite the more common availability of body powered elbow joints [40]. Being able to articulate a wrist non-passively is especially important for bilateral amputees, who have difficulty articulating a passive wrist by external forces, particularly a multi-DOF system. Body powered actuation is incorporated into wrists in two ways: either to toggle the locking of passive joints (by similar methods to those previously described in Section IV.A) or to provide direct joint actuation.

\section{1) Body Powered Single Rotators}

Examples of passive pronation joints with body powered locking mechanisms are given in [15], [29] and the HD 4Function Wrist [15] (Fig 3.e.) of both of which also have an elastic return to a neutral position when unlocked. In [41] tension applied to the body powered cable actuates a pronation joint. When the tension is released, the wrist locks, and switches the direction of rotation for the subsequent cable pull. 


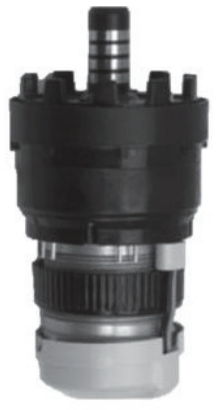

(a) $1 R$

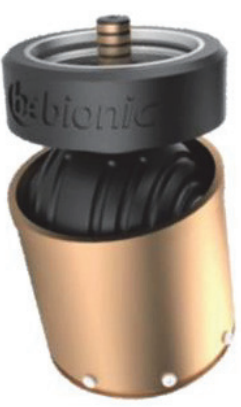

(b) S

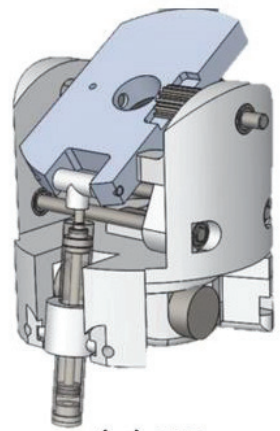

(c) $2 \mathrm{R}$

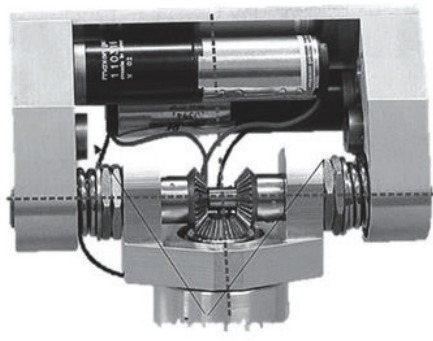

(d) $2 R$

Fig. 2. Active Wrist Mechanisms. (a) Otto Bock Wrist Rotator (1R) [24], (b) Bebionic Concept Wrist (S) [67], (c) Roose Pneumatically Powered Wrist Prosthesis (2R) [561. (d) Kvberd et al. 2-DOF Powered Prosthetic Wrist 「571.

\section{2) Body Powered $2 R$}

In [42], locks on passive pronation and flexion DOF may be independently toggled via two separate Bowden cables. Of course, each additional cable requires its own routing and harnessing, as well as a unique motion for the amputee to operate it. This can make the harnessing and the control of multiple cable devices complex and problematic.

\section{Active Wrist Prostheses}

Active wrist prostheses utilize electromechanical actuators and a power source to achieve motion at the wrist. Active devices are typically controlled via myoelectric methods, where electrodes in contact with the user's skin provide control inputs to electromechanical actuators. Active wrist prostheses may be modular standalone units, compatible with a variety of TDs, (e.g. [24],[43]) or may already be integrated into TD or more proximal (e.g. transhumeral) arm prosthesis [44]-[47].

1) Active Single DOF

Active single DOF prostheses are available as pronation [24], [43], [48]-[52] or flexion [21], [53], [54] devices.

\section{a) Rotators}

Commercial standalone units such as the MC Electric Rotator [43] and the OB Wrist Rotator [24] (Fig. 4.a.) are designed to be compatible with many TDs, leading to widespread acceptance. A non-commercial standalone device described in [49] implemented pronation at an angle skewed to the forearm longitudinal axis under the impression that rotation about this axis was more useful than the standard pronation axis.

Powered rotators do not share the same compactness as their passive counterparts. This results in length issues discussed in Section III.D. The OB Michelangelo Hand [48] utilizes a wrist rotator which partially fits within the body of the hand itself, reducing overall length. Other TDs with incorporated active wrist rotators include [50], [51], of which the latter is the MANUS hand, pictured in fig. $5 \mathrm{a}$.

\section{b) Flexors}

Single DOF flexion wrists may be fabricated as part of powered hands. In [21], [53] flexion actuators are housed within the body of the hand, reducing overall length but increasing distal weight. In [54] the flexion actuator is housed in the forearm socket.

\section{2) Active $2 R$ Wrist}

These 2 DOF mechanisms are focus of much work in the prosthetics research community. Once again, some active standalone $2 \mathrm{R}$ wrists are combinations of $1 \mathrm{R}$ units mounted in series at $90^{\circ}$ offsets [55], resulting in a relatively long serial chain and issues discussed in Section III.D.

The wrist described in [56] (fig. 4c) implements pronation and flexion in a compact unit suitable for users with distal amputations. The placement and orientation of actuators reduces the requirement for forearm socket space. Similarly, in [57] (fig. 4d), actuators are placed distal to the actual wrist joint in a compact design via a differential transmission. This allows the motors to be placed obliquely to the axis to the forearm, and external to the socket, similar to in integrated hands highlighted in Section VI.1)b). The distal hardware arrangement makes the system suitable for wrist disarticulation amputates (see Section III.D), though a distal weight offset will result. Other transradial prostheses that incorporate active $2 \mathrm{R}$ wrists include [58]-[64], all of which house actuators within the forearm.

Transhumeral prostheses with $2 \mathrm{R}$ wrists [44]-[47] can fully utilize the forearm space and successfully implement wrists with 2 DOF. The DEKA Arm [46] and the RIC Arm [47] are two notable prostheses currently undergoing development. The RIC Arm features wrist flexion and both incorporate wrist rotation, though the DEKA Arm uses coupled flexion and radial deviation as its second DOF.

\section{3) Active $3 R$ Wrists}

These mechanisms offer 3 powered DOF motion. In [65], the Modular Prosthetic Limb (MPL, fig. 5b) pronation is achieved via a wrist rotator in the proximal forearm. Following this, a chain of 2 identical motors which have orthogonal axes are placed in series with the rotator. In [66], a 3 DOF wrist is achieved by stacking 3 servo motors at right angles with one another.

\section{4) Active Spherical Wrist}

Although $\mathrm{S}$ joints have 3DOF (Fig. 2.e.), the only active wrist making use of an $\mathrm{S}$ joint reduces the joint to 2DOF. In the RSL Steeper's Bebionic Concept Wrist (fig. 4b) [67] motion is prevented in the radial deviation DOF. By 


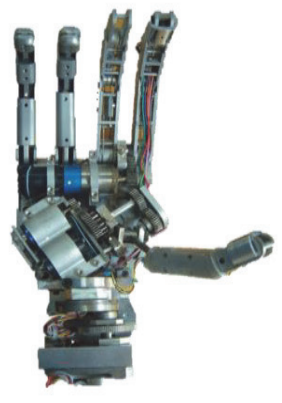

(a) $1 \mathrm{R}$

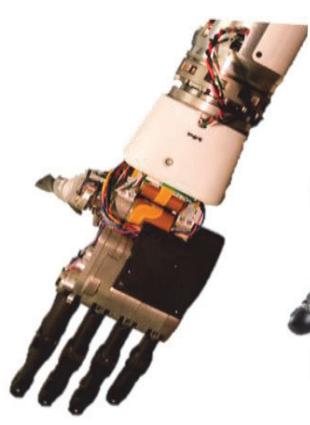

(b) $2 \mathrm{R}$

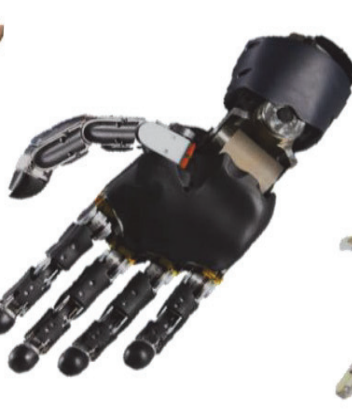

(b) $3 R$

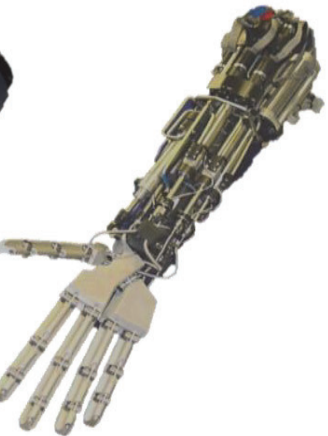

(c) $1 \mathrm{R}, 1 \mathrm{RPR}, 1 \mathrm{SPS}$

Fig. 5. Active Wrist Prostheses as part of anthropomorphic TDs, (a) MANUS Hand (R) [51], (b) DEKA Arm (2R) [46], (c) Modular Prosthetic Limb (3R) [65], (c) Vanderbilt gas powered transhumeral prosthesis (1R, 1RPR 1SPS) [70].

engaging pins into grooves on the sphere, a user may select actuated motion in either pronation or flexion DOF. These are powered by the same actuator. Unlike the passive LTI OmniWrist (Section IV.B.2), pronation is enabled, so the reduced $\mathrm{S}$ joint does not behave as a $\mathrm{U}$ joint.

\section{5) Active RS Wrists}

RS mechanisms theoretically have more DOFs than the human wrist, but in the implementation realized in [68], the TD was constrained not to pronate (roll) via the $\mathrm{S}$ joint. Instead pronation was achieved by a proximal rotator.

\section{6) Active Parallel Wrist}

Only a single wrist prosthesis has been designed as a parallel mechanism. The wrist detailed in [69] utilizes 5 proximally actuated tendons to control the orientation of a plate fixed to a spherical joint. The authors consider each tendon an SPS serial chain, making the complete wrist a 5SPS, 1S mechanism. An analogous central 1S structure is shown for a parallel robotic wrist in Fig 6. Actuators placement was not addressed in the publication.

\section{7) Hybrid mechanisms}

The hybrid mechanisms based wrists of [70]-[72], are composed of an $\mathrm{R}$ joint serving pronation, followed by a 2 DOF parallel mechanism to provide flexion and radial deviation. In [70] (fig. 5c), the flexion and radial deviation DOF are actuated by prismatic linear actuators in a 1-RPR 1-SPS parallel mechanism. To achieve pronation, a linear screw rod rotates the entire parallel mechanism. All hybrid examples are part of transhumeral prostheses that utilize the entire forearm to accommodate mechanics and actuation.

\section{DISCUSSION}

This review of wrist prostheses has identified various themes and issues present in existing technology and in turn has identified areas for improvement in future devices (Table 1 summarizes the mechanical design specifications of some of the wrists discussed in previous sections, when those specs have been made available). Looking across the spectrum, a few things stand out. In terms of commercial availability, most devices are passive, generally either 1 - or 2-DOF devices, with a few powered rotators available. Indeed, the majority of wrist designs of all types included pronation capability, which is a highly sought function by upper-limb amputees [73] with flexion is the second most requested DOF, followed by radial deviation [73]. The majority of multi DOF wrists are serial mechanisms, which, while simple to design and manufacture, are more prone to excessive length. Alternatively, parallel mechanisms, which are used extensively in robotics, may be an area for future development of new wrist prostheses (e.g. Fig.6 [74]).

\section{A. Passive Wrists}

Single DOF passive wrists are simple devices that can implement simple, manual articulation in compact and lightweight packages. Friction clutches and locking features (Section III.A) add stability and significant additional functionality to an articulating wrist. Articulation of the wrist by these methods mainly falls on the contralateral hand, for repositioning and locking / unlocking. Though the body and environmental features may be used when the contralateral hand is absent or occupied [14], such an interface is less than ideal (Section III.D).

Higher DOF in passive wrists is often achieved by stacking multiple single DOF units. This negates the compactness of the individual units. Some wrists circumvent this problem by utilizing higher mobility $\mathrm{S}$ and U joints [23], [24], [37] (Section IV.B.2). Passive devices with elastic returns (e.g. [33] [35]) can impart some additional functionality without requiring the opposite hand for articulation (Section III.A). Of course, elastic elements are primarily applicable to particular classes of tasks, as previously discussed in Section IV.A.

\section{B. Body Powered Wrists}

There is a distinct lack of body powered prosthetic wrists, despite general popularity of body powered terminal devices and elbow joints. Unlike the common use of body power for articulation in TDs, some body powered wrist interfaces serve only to toggle a position locking mechanism [15],[15], [29], relying again on the contralateral hand for re-positioning. Body powered wrist articulation was indicated as highly desirable by trans-radial users of body powered systems [73]. As such, there is much room and potential for body powered wrist interfaces that permit articulation. 
TABLE 1. WRIST SPECIFICATIONS

\begin{tabular}{|c|c|c|c|c|c|c|c|c|}
\hline Product Name / Lead Author & Commercial & DOF $^{\mathrm{a}}$ & Actuation & Configuration & Diameter (cm) & Length (cm) & Weight (g) & Stall Torque (Nmm) \\
\hline Plettenburg, D. H. [19] & $\mathrm{N}$ & $\mathrm{P}$ & Passive & \begin{tabular}{|l|}
$\mathrm{R}$ \\
\end{tabular} & $3-3.8$ & - & $12-20$ & N/A \\
\hline Kesheng Rotator [21] & $\mathrm{Y}$ & $\mathrm{P}$ & Passive & $\mathrm{R}$ & 4.7 & 4.5 & - & $\mathrm{N} / \mathrm{A}$ \\
\hline Hosmer Rotator [15] & $\mathrm{Y}$ & $\mathrm{P}$ & Passive & $\mathrm{R}$ & 5.0 & - & - & N/A \\
\hline Hosmer Sierra Wrist [15] & $\mathrm{Y}$ & $\mathrm{F}$ & Passive & $\mathrm{R}$ & 5.0 & 3.6 & 113 & N/A \\
\hline MC Flexion Wrist [33] & $\mathrm{Y}$ & $\mathrm{F}$ & Passive & RR & -- & 4.2 & 55 & $\mathrm{~N} / \mathrm{A}$ \\
\hline Montagnani, F. [35] & $\mathrm{N}$ & $\mathrm{F}+\mathrm{A}$ & Passive & $\mathrm{RR}$ & 9.5 & 6.5 & 450 & N/A \\
\hline MC MultiFlex [37] & $\mathrm{Y}$ & $\mathrm{F}+\mathrm{P}$ & Passive & RRR & -- & 4.8 & 83 & N/A \\
\hline OB MyoLino [24] & $\mathrm{Y}$ & $\mathrm{F}+\mathrm{A}$ & Passive & $\mathrm{S}$ & 4.0 & 3.9 & 49.4 & $\mathrm{~N} / \mathrm{A}$ \\
\hline Kesheng Flexor [21] & $\mathrm{Y}$ & $\mathrm{F}$ & Active & $\mathrm{R}$ & 4.7 & 5.1 & - & -- \\
\hline OB Wrist Rotator [24] & $\mathrm{Y}$ & $\mathrm{P}$ & Active & $\mathrm{R}$ & -- & - & 96 & -- \\
\hline MC Electric Rotator [43] & $\mathrm{Y}$ & $\mathrm{P}$ & Active & $\mathrm{R}$ & 4.7 & 7.0 & 143 & -- \\
\hline Kato, I. [54] & $\mathrm{N}$ & $\mathrm{F}$ & Active & $\mathrm{R}$ & 5.6 & -- & - & -- \\
\hline Zinck, A. [49] & $\mathrm{N}$ & $\mathrm{P}$ & Active & $\mathrm{R}$ & 4.0 & 6.5 & 87 & 60 \\
\hline Abd Razak, N. A. [55] & $\mathrm{N}$ & $\mathrm{P}+\mathrm{F}$ & Active & RR & -- & -- & 500 & 13000 \\
\hline Controzzi, M.[59] & $\mathrm{N}$ & $\mathrm{P}+\mathrm{F}$ & Active & $\mathrm{RR}$ & -- & -- & $240^{\mathrm{b}}$ & -- \\
\hline Kyberd, P. J. [57] & $\mathrm{N}$ & $\mathrm{P}+\mathrm{F}$ & Active & RR & 9.6 & 5.0 & 200 & 73 \\
\hline Roose, C. [56] & $\mathrm{N}$ & $\mathrm{P}+\mathrm{F}$ & Active & RR & 5.3 & -- & 95 & $321 \mathrm{Fl}$ \\
\hline RIC Arm [47] & $\mathrm{N}$ & $\mathrm{P}+\mathrm{F}$ & Active & $\mathrm{RR}$ & -- & -- & $170 / 155$ & $900 / 1000$ \\
\hline Mahmoud, R. [66] & $\mathrm{N}$ & $\mathrm{P}+\mathrm{F}+\mathrm{A}$ & Active & RRR & -- & -- & -- & $930 / 210 / 210$ \\
\hline RSL Steeper BeBionic Wrist [67] & $\mathrm{Y}$ & $\mathrm{P}+\mathrm{F}$ & Active & $\mathrm{S}$ & 5.0 & 7.5 & -- & -- \\
\hline
\end{tabular}

\section{Active Wrists}

Within active wrists, the most apparent observation is the scarcity of 3 DOF compact powered wrist devices. The hybrid mechanisms which offer 3 DOF wrist motion are not compact, and are designed to occupy the entirety of the forearm volume, greatly limiting applicability (Section VI.7)). However, the parallel wrist in [69] permitted a compact 3 DOF mechanism, though actuator placement had yet to be addressed.

Unlike passive wrists, some higher DOF active wrists use more integrated approaches to achieve multi DOF motion, either by reducing packaging between elements, placing actuators in the hand [21], [48], [53], [57], using higher mobility joints [67]-[69] or alternative drive mechanisms such as differentials [57], [59]. However, such higher DOF active wrists often incur a tradeoff between size/weight and torque capabilities. Active wrists which can achieve torques similar to the healthy human wrist [13] can be rather heavy or long [47], [55], [66]. Relatively compact active wrists have very low torque [49], [56], [57]. This tradeoff must be addressed in the development of more sophisticated wrist prostheses. At the time of writing this review, no multi DOF active wrists are commercially available, though some are known to be in development.

\section{REFERENCES}

[1] K. Ziegler-Graham, E. J. MacKenzie, P. L. Ephraim, T. G. Travison, and R. Brookmeyer, "Estimating the prevalence of limb loss in the United States: 2005 to 2050," Arch. Phys. Med. Rehabil., vol. 89, no. March, pp. 422-429, 2008

[2] B. D. Adams, N. M. Grosland, D. M. Murphy, and M. McCullough, "Impact of impaired wrist motion on hand and upper-extremity performance," J. Hand Surg. Am., vol. 28, no. 6, pp. 898-903, Nov. 2003.

[3] A. G. Mell, B. L. Childress, and R. E. Hughes, "The effect of wearing a wrist splint on shoulder kinematics during object manipulation," Arch. Phys. Med. Rehabil., vol. 86, no. 8, pp. 1661-1664, 2005.
[4] F. Montagnani, M. Controzzi, and C. Cipriani, "Is it finger or wrist dexterity that is missing in current hand prostheses?," IEEE Trans. Neural Syst. Rehabil. Eng., vol. 21, no. c, pp. 1-1, 2015.

[5] D. C. Boone and S. P. Azen, "Normal range of motion of joints in male subjects.," J. Bone Joint Surg. Am., vol. 61, pp. 756-759, 1979.

[6] M. M. Marshall, J. R. Mozrall, and J. E. Shealy, "The effects of complex wrist and forearm posture on wrist range of motion.," Hum. Factors, vol. 41, no. 2, pp. 205-213, 1999.

[7] J. M. Soucie, C. Wang, a. Forsyth, S. Funk, M. Denny, K. E. Roach, and D. Boone, "Range of motion measurements: Reference values and a database for comparison studies," Haemophilia, vol. 17, pp. 500-507, 2011.

[8] D. L. Nelson, M. A. Mitchell, P. G. Groszewskv, S. L. Pennick, and P. R. Manske, "Wrist range of motion in activities of daily living," no. Table 1, pp. 329-334, 1994.

[9] R. H. Brumfield and J. a Champoux, "A biomechanical study of normal functional wrist motion.," Clinical orthopaedics and related research. pp. 23-25.

[10] M. Sardelli, R. Z. Tashjian, and B. a MacWilliams, "Functional elbow range of motion for contemporary tasks.," J. Bone Joint Surg. Am., vol. 93, pp. 471-477, 2011

[11] J. Ryu, W. P. C. Iii, L. J. Askew, K. An, and E. Y. S. Chao, "Functional ranges of motion of the wrist joint," pp. 409-419, 1991.

[12] W. N. Timm, S. W. O’Driscoll, M. E. Johnson, and K. N. An, "Functional comparison of pronation and supination strengths.," J. Hand Ther., vol. 6, no. 3, pp. 190-193.

[13] J. L. Morse, M. C. Jung, G. R. Bashford, and M. S. Hallbeck, "Maximal dynamic grip force and wrist torque: The effects of gender, exertion direction, angular velocity, and wrist angle," Appl. Ergon., vol. 37, pp. 737-742, 2006

[14] G. Principles, "Special considerations: fitting and training the bilateral

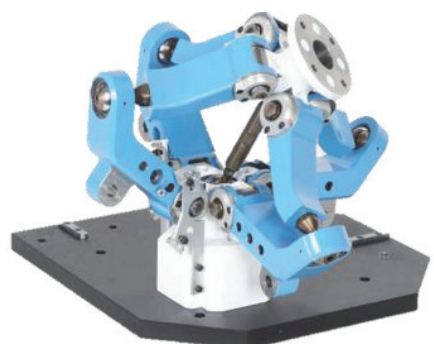

Fig. 6. A parallel mechanism, the Ross-Hime Omni-Wrist (not to be confused with the LTI OmniWrist). [74] 
upper-limb amputee," pp. 1-12, 2011.

[15] "The Hosmer Dorrance Corporation." [Online]. Available: http://hosmer.com/.

[16] "PKM Tricept.” [Online]. Available: http://www.pkmtricept.com/.

[17] E. a Biddiss and T. T. Chau, "Upper limb prosthesis use and abandonment: a survey of the last 25 years.," Prosthet. Orthot. Int., vol. 31, no. 3, pp. 236-257, 2007.

[18] W. C. Prout, "Prosthetic wrist unit," 3159847, 1965.

[19] D. H. Plettenburg, "The WILMER passive hand prosthesis for toddlers," JPO J. Prosthetics Orthot., vol. 21, pp. 97-99, 2009.

[20] S. C. Jacobsen, "Electrically driven artificial arm," US4521924, 1985.

[21] "Shanghai Kesheng Prosthese Co., LTD." [Online]. Available: http://www.keshen.com/.

[22] D. R. W. May, "Prosthetic wrist fitting," 4156945, 1979.

[23] "Liberating Technologies, Inc." [Online]. Available: http://www.liberatingtech.com/.

[24] "Otto Bock." [Online]. Available: http://www.ottobock.com/.

[25] E. Horvarth, "Artificial wrist and arm prosthesis," US4010495, 1977.

[26] J. K. Northrop, "Prosthetic wrist," US2457316, 1946.

[27] G. M. Motis, "Artificial arm with stepped up wrist drive and automatic wrist lock," 2638604, 1953.

[28] K. Vesper, "Wrist mechanism for artificial arms," 2605476, 1952.

[29] W. C. Prout, "Universal wrist system," 3798680, 1974.

[30] G. M. Motis, "Wrist flexion unit," 2692390, 1954.

[31] T. Bertels, "Verriegelbares prothesengelenk," EP1852092A1, 2007.

[32] T. Bertels, "Biomechanic aspects and patient needs lead the path to a unique wrist joint for myoelectric prosthesis," pp. 17-20, 2005.

[33] A. D.- Dyck, S. R. Kunz, L. City, J. R. Linder, W. Jordan, H. Harold, S. L. City, R. Cited, S. L. City, and P. E. H. Willse, "Wrist devive for use with a prosthetic limb," 2006.

[34] M. W. Razink, "Prosthetic wrist," US8795387, 2014.

[35] F. Montagnani, M. Controzzi, C. Cipriani, and S. Member, "Preliminary design and development of a two degrees of freedom passive compliant prosthetic wrist with switchable stiffness," in ROBIO, 2013, no. December, pp. 310-315

[36] N. Dechev, W. L. Cleghorn, and S. Naumann, "Multiple finger, passive adaptive grasp prosthetic hand," Mech. Mach. Theory, vol. 36, pp. 1157 $1173,2001$.

[37] S. Archer, A. Dyck, R. Grant, E. Iversen, J. Jcobs, S. Kunz, J. Linder, and H. Sears, "Wrist device with use for a prosthetic limb," US2007/0173955, 2007.

[38] K. J. De Laurentis and C. Mavroidis, "Mechanical design of a shape memory alloy actuated prosthetic hand," Technol. Heal. Care, vol. 10, pp. 91-106, 2002.

[39] B. D. Veatch and J. D. Scott, "Pre-positionable prosthetic hand," 0188952, 2008.

[40] S. C. Jacobsen and D. F. Knutti, "Body-powered prosthetic arm," US5888235, 1999.

[41] G. M. Motis, "Linear to rotational movement converter," 3446937, 1969.

[42] J. H. Rouse, R. H. Farquharson, and C. G. Betts, "Multifunction body powered prosthetic wrist unit and method," 7048768, 2003.

[43] "Motion Control Inc." [Online]. Available: http://www.utaharm.com/.

[44] a. S. Poulton, P. J. Kyberd, and D. Gow, "Progress of a modular prosthetic arm," 2002.

[45] D. J. Gow, "Upper limb prosthesis,” 6361570, 2002.

[46] L. Resnik, S. L. Klinger, and K. Etter, "The DEKA Arm: Its features, functionality, and evolution during the Veterans Affairs Study to optimize the DEKA Arm.," Prosthet. Orthot. Int., p. $0309364613506913-, 2013$.

[47] J. Sensinger, J. Lipsey, T. Sharkey, A. Thomas, L. Miller, K. Turner, J. Ochoa, and T. Idstein, "Initial experiences with the RIC arm," in Myoelectric Controls Symposium, 2014.

[48] G. Puchhammer, "Michelangelo Hand (Articulated Hand Prosthesis)," US8690963, 2014.

[49] A. Zinck, Ø. Stavdahl, E. Biden, and P. J. Kyberd, "Design of a compact, reconfigurable, prosthetic wrist," Appl. Bionics Biomech., vol. 9, no. 1, pp. 117-124, 2012.

[50] J. Zajdlik, "The preliminary design and motion control of a Five-fingered prosthetic hand," 2006 Int. Conf. Intell. Eng. Syst., vol. 2, pp. 202-206, 2006 .
[51] J. L. Pons, E. Rocon, R. Ceres, D. Reynaerts, B. Saro, S. Levin, and W. Van Moorleghem, "The MANUS-HAND dextrous robotics upper limb prosthesis: Mechanical and Manipulation Aspects," Auton. Robots, vol. 16, pp. 143-163, 2004.

[52] M. Troncossi, E. Gruppioni, M. Chiossi, A. G. Cutti, A. Davalli, and V. Parenti-Castelli, "A novel electromechanical shoulder articulation for upper-limb prostheses: From the Design to the First Clinical Application," JPO J. Prosthetics Orthot., vol. 21, pp. 79-90, 2009.

[53] G. Rennerfelt, "Artificial hand," US4792338, 1988.

[54] I. Kato, S. Yamakawa, K. Ichikawa, and M. Sano, "Multifunction myoelectric hand prosthesis with pressure sensory feedback system waseda hand 4p," in Adances in the External Control of Human Extremities, 1963.

[55] N. A. Abd Razak, N. A. Abu Osman, H. Gholizadeh, and S. Ali, "Development and performance of a new prosthesis system using ultrasonic sensor for wrist movements: a preliminary study.," Biomed. Eng. Online, vol. 13, p. 49, 2014

[56] C. Roose, "Pneumatically powered wrist prosthesis," 2014

[57] P. J. Kyberd, E. D. Lemaire, E. Scheme, C. MacPhail, L. Goudreau, G. Bush, and M. Brookeshaw, "Two-degree-of-freedom powered prosthetic wrist," J. Rehabil. Res. Dev., vol. 48, no. 6, p. 609, 2011.

[58] H. L. and F. Mohl, "An electromechanical forearm and hand," in Adances in the Control of Human Extremities.

[59] M. Controzzi, C. Cipriani, B. Jehenne, M. Donati, and M. C. Carrozza, "Bio-inspired mechanical design of a tendon-driven dexterous prosthetic hand," 2010 Annu. Int. Conf. IEEE Eng. Med. Biol. Soc. EMBC'10, no. i, pp. 499-502, 2010.

[60] S. L. Phillips, K. J. De Lauretis, and C. E. I. Pfeiffer, "Joint Prosthetic Device," 0326677, 2009.

[61] H. Takeda, N. Tsujiuchi, T. Koizumi, H. Kan, M. Hirano, and Y. Nakamura, "Development of prosthetic arm with pneumatic prosthetic hand and tendon-driven wrist," Proc. 31st Annu. Int. Conf. IEEE Eng. Med. Biol. Soc. Eng. Futur. Biomed. EMBC 2009, pp. 5048-5051, 2009.

$[62]$ K. Ito, T. Tsuji, A. Kato, and M. Ito, "An EMG controlled prosthetic forearm in three degrees of freedom using ultrasonic motors," 1992 14th Annu. Int. Conf. IEEE Eng. Med. Biol. Soc., vol. 4, pp. 1487-1488, 1992.

[63] K. Ohnishi, T. Morio, T. Takagi, and I. Kajitani, "Multimodal sensor controlled three Degree of Freedom transradial prosthesis," IEEE Int. Conf. Rehabil. Robot., 2013.

[64] R. A. Frosch, G. A. Wiker, and W. A. Mann, "Compact artificial hand," 4149278,1979

[65] M. S. Johannes, J. D. Bigelow, J. M. Burck, S. D. Harshbarger, M. V. Kozlowski, and T. Van Doren, "An overview of the developmental process for the modular prosthetic limb," Johns Hopkins APL Tech. Dig. (Applied Phys. Lab., vol. 30, no. 3, pp. 207-216, 2011.

[66] R. Mahmoud, A. Ueno, and S. Tatsumi, "Dexterous mechanism design for an anthropomorphic artificial hand: Osaka City University Hand I," 2010 10th IEEE-RAS Int. Conf. Humanoid Robot. Humanoids 2010, pp. 180$185,2010$.

[67] E. Varley, “An artificial joint,” GB2012000030.

[68] G. T. Pinson, "Digitally-controlled artificial hand," 4246661, 1981

[69] S. K. Mustafa, G. Yang, S. H. Yeo, W. Lin, and C. B. Pham, "Development of a bio-inspired wrist prosthesis," 2006 IEEE Conf. Robot. Autom. Mechatronics, vol. 1, pp. 3-8, 2006

[70] K. B. Fite, T. J. Withrow, X. Shen, K. W. Wait, J. E. Mitchell, and M. Goldfarb, "A gas-actuated anthropomorphic prosthesis for transhumeral amputees," IEEE Trans. Robot., vol. 24, no. 1, pp. 159-169, 2008.

[71] S. K. Kundu and K. Kiguchi, "Development of a 5 DOF prosthetic arm for above elbow amputees," Proc. 2008 IEEE Int. Conf. Mechatronics Autom. ICMA 2008, pp. 207-212, 2008

[72] D. S. V. Bandara, R. A. R. C. Gopura, K. T. M. U. Hemapala, and K Kiguchi, "A multi-DoF anthropomorphic transradial prosthetic arm," in BIOROB, 2014, vol. 1, pp. 1039-1044.

[73] D. J. Atkins, D. C. Y. Heard, and W. H. Donovan, "Epidemiologic overview of individuals with upper-limb loss and their reported research priorities," JPO J. Prosthetics Orthot., vol. 8, no. 5, p. 2-11, 2015.

[74] M. E. Rosheim, "Robotic Manipulator," 5979264, 1999. 